\title{
Analysis of the possibility of using a video camera as a UAV sensor
}

\author{
Rafał Dąbrowski ${ }^{a}$, Paulina Deliśa ${ }^{\mathrm{a}}$, Marek Wyszyński ${ }^{\mathrm{b}}$ \\ ${ }^{\mathrm{a}}$ Department of Remote Sensing and Photogrammetry, ${ }^{\mathrm{b}}$ Department of Geodesy, \\ Instytut Geo Institute of Geodesy, Faculty of Civil Engineering and Geodesy, \\ Military University of Technology ul. Gen. Sylvester Kaliski 2, 00-908 Warsaw 49, Poland
}

\begin{abstract}
In the ISPRS final report from 2012 it was accented that light and low cost Unmanned Aerial Vehicles are playing a more and more important role when it comes to carrying remote sensing and photogrammetric sensors. Such platforms are characterized by a small weight, low cost of purchase and later exploitation and, depending on their technical specifications, a payload of about 1,5 kg. The above characteristics make these platforms an attractive alternative for carrying sensors in comparison to a traditional airplane, especially whilst conducting photogrammetric and remote sensing studies of small areas.

However because of the size and mass of such UAV's, data acquired by means of the sensors which they carry is characterized by very dynamically changing in time exterior orientation parameters. In extreme cases this can cause no forward overlap between subsequent frames which makes such data, in most circumstances, useless.

An interesting solution is using a video camera (or a number of video cameras) as a sensor. Such cameras enable, depending on the standard of registration, the acquisition of tens of images every second, which means a very large forward overlap.

The article contains the analyses of the possibility of using the FCO HD 1080i and FCO HD 720i video cameras as a UAV sensor. Each of the analyzed cameras is different in terms of their build, as well as the quality of the acquired imagery, however that all have the same low weight. An evaluation was made in two different aspects: geometrical and photographic.

Based on specialized test fields it was possible to determine the exterior orientation parameters ofthese cameras which allowed for an analysis of their invariability. Other parameters which had been determined and analyzed include: the spatial resolution, the way in which colours are registered and aberrations which were present within the optics of these cameras.
\end{abstract}

Keywords: video camera, camera calibration, UAV.

\section{Introduction}

Whilst observing modern trends in developments in photogrammetry and remote sensing it is not possible to overlook the growing trend in adapting light unmanned aerial vehicles (UAV) as platforms assigned to collect data. The quality of received data (which is directly linked to the accuracy of the final product upon which they had been created) is determined by many factors [1]. The most important of them include: the stability of the flight path of the platform, the flight altitude, and the quality of the sensor itself. The quality of the system is determined by the spatial and radiometric resolution of the image and aberrations with which they are burdened [2].

Digital cameras are most commonly used as a data collecting sensor in "cheap" UAV. These are equipped with a lens with a constant focal length $\left(\mathrm{C}_{\mathrm{K}}\right)$ and a remote trigger option.

However the use of a frame sensor creates a few crucial problems e.g.: the occurrence of sets of images with an irregular geometry (irregular along-track overlap) and the possibility to collect data only in the visible range of the EM spectrum.

The problems above are arising primarily due to dynamically changeable in time exterior orientation parameters and also by problems connected directly with the sensor. A striking example is the issue of the speed of recording a large number of images in quick succession. In this situation, most cameras are not able to save the acquired imagery onto a card even with a large buffer which leads to pauses in the camera's readiness to acquire new imagery. This delay can take up to several seconds.

In order to prevent the above problems one can seek to minimize the flight speed of the platform or to record the external orientation parameters (more expensive UAV systems).

Corresponding author: Rafał Dabrowski. E-mail address: dabrowski@wat.edu.pl

http://dx.doi.org/10.3846/enviro.2014.200

(C) 2014 The Authors. Published by VGTU Press. This is an open-access article distributed under the terms of the Creative Commons Attribution License, which permits unrestricted use, distribution, and reproduction in any medium, provided the original author and source are credited. 
An interesting solution to minimize these problems is to use a video camera imaging sensor. Despite recording a lower resolution image, the video technique allows, depending on the data recording standard, for the acquisition of tens of images per second, which translates into a series of images with a large along-track coverage (high number of additional observations). A particularly intriguing problem is the adaptation of amateur lightweight video cameras for Photogrammetry.

This paper will assess the possibility of using FCO HD 1080i and FCO HD 720i cameras as an imaging sensor installed on-board a UAV. This evaluation will be made on the basis of the calibration process of these cameras both in terms of their geometric and photographic quality. The camera calibration is in fact a process in which certain characteristics are determined describing the quality of the images acquired by the given camera [3].

The procedures for accurate camera calibration and image orientation are a necessary first step in the process of extracting precise and reliable $3 \mathrm{D}$ data from the image $[4,5,6]$.

\section{Characteristics of the HD $1080 \mathrm{P}$ and FCO HD $720 \mathrm{P}$ video cameras}

Two ultra-light video cameras from the Flycam family were selected for this research: Flycam One HD 720 p and Flycam One HD 1080 p. These cameras acquire image data in HD and are characterized by a low weight. Selected specifications of both cameras are listed in Table 1.

Table 1. Technical parameters of FCO HD 1080P and FCO HD 720

\begin{tabular}{lll}
\hline Video Camera & Fly Cam One HD $1080 \mathrm{p}$ & Fly Cam One HD 720 p \\
\hline Sensor resolution $[\mathrm{Px}]$ & $1920 \times 1080$ & $1280 \times 720$ \\
Converter & $5 \mathrm{Mpi} \times \mathrm{CMOS}$ & $5 \mathrm{Mpi} \times$ CMOS \\
Size $[\mathrm{mm}]$ & $95 \times 50 \times 19 \mathrm{~mm}$ & $95 \times 42 \times 19 \mathrm{~mm}$ \\
Weight $[\mathrm{g}]$ & 90 & 59 \\
Data recording & Flashcard MPEG4 & Flashcard MPEG4 \\
Operating temperature $\left[{ }^{\circ} \mathrm{C}\right]$ & -10 do 50 & -10 do 60 \\
Power supply & USB: $5 \mathrm{~V} /$ port $10-\mathrm{pin}$, & USB: $5 \mathrm{~V} /$ port $10-\mathrm{pin}$, \\
& batery LiIon: $3,7 \mathrm{~V} 900 \mathrm{mAh}$ & LiPo: $3,7 \mathrm{~V} 600 \mathrm{mAh}$ \\
\hline
\end{tabular}

\section{Geometric aspect}

In the process of calibrating these cameras, the first aspect which will determine their suitability as a UAV sensor is their geometric quality. This aspect is related to an analytical determination of the geometric parameters which have a disrupting affect on the image registration process.

The geometric calibration had been carried out on the basis of a specialized 2D test field. The acquisition of imaging data consisted of the registration of 12 sequence series made in five configurations of the position of the sensor relative to the test. After each sequence, the camera was switched off and turned on again. Exposure parameters were set optimally to the lighting conditions, and uniform during the whole duration of the measurements.

Processing of the acquired image data had been conducted on the basis of algorithms implemented in PhotoModeler Scaner (PMS) and Topcon Image Master Calib (TimC) software.

The procedures implemented in PMS and TIMC differ from each other.

The PMS software provides a fully automatic calibration without any user interaction. TICM software requires the user to enter an approximate value of the focal length and to manually select 4 defined points of the test field on the image.

As a result of this image data processing the following parameters are defined: the focal length, the coordinates of the PPA, the coefficients of the radial distortion. The number of obtained parameters allowed us to check their consistency over time (successive cycles preceded by camera ON and camera OFF). The results obtained using the PhotoModeler Scanner software for each camera are shown in Table 2 and 3.

Table 2. Inner orientation parameters Fly Cam One HD 1080P

\begin{tabular}{cccccccc}
\hline & $\mathbf{c}_{\mathbf{k}}[\mathbf{m m}]$ & $\mathbf{x}_{\mathbf{0}}[\mathbf{m m}]$ & $\mathbf{y}_{\mathbf{0}}[\mathbf{m m}]$ & $\mathbf{k}_{\mathbf{1}}$ & $\mathbf{k}_{\mathbf{2}}$ & $\mathbf{p}_{\mathbf{1}}$ & $\mathbf{p}_{\mathbf{2}}$ \\
\hline $\bar{x}$ & 10.693 & 2.965 & 1.600 & 0.002064 & -0.000099 & 0.000076 & -0.000350 \\
$\boldsymbol{\sigma}$ & 0.016 & 0.040 & 0.009 & 0.000049 & 0.000006 & 0.000031 & 0.000046 \\
Min & 10.674 & 2.917 & 1.586 & 0.002002 & -0.000109 & 0.0000390 & -0.000408 \\
Max & 10.713 & 3.008 & 1.608 & 0.002118 & -0.000093 & 0.0001050 & -0.000295 \\
Med. & 10.693 & 2.967 & 1.603 & 0.002069 & -0.000098 & 0.000080 & -0.000347 \\
\hline
\end{tabular}


Table 3. Inner orientation parameters Fly Cam One HD 720P

\begin{tabular}{cccccccc}
\hline & $\mathbf{c}_{\mathbf{k}}[\mathbf{m m}]$ & $\mathbf{x}_{\mathbf{0}}[\mathbf{m m}]$ & $\mathbf{y}_{\mathbf{0}}[\mathbf{m m}]$ & $\mathbf{k}_{\mathbf{1}}$ & $\mathbf{k}_{\mathbf{2}}$ & $\mathbf{p}_{\mathbf{1}}$ & $\mathbf{p}_{\mathbf{2}}$ \\
\hline $\bar{x}$ & 7.980 & 3.003 & 1.661 & 0.003072 & -0.000292 & 0.000076 & -0.00035 \\
$\boldsymbol{\sigma}$ & 0.030 & 0.008 & 0.030 & 0.000049 & 0.000260 & 0.000067 & 0.000078 \\
Min & 7.959 & 2.993 & 1.621 & 0.003023 & -0.000683 & 0.000204 & -0.000165 \\
Max & 8.025 & 3.012 & 1.691 & 0.003137 & -0.000146 & 0.000338 & 0.000021 \\
Med. & 7.968 & 3.004 & 1.666 & 0.003065 & -0.000160 & 0.000286 & -0.000055 \\
\hline
\end{tabular}

The FCO HD 1080p camera has a focal length value of $10,693 \mathrm{~mm}$ and a pixel dimension $(3,1 \mathrm{um} \times 3,1 \mathrm{um})$, while the FCO HD 720p camera respectively 7,980 mm and $4.6 \times 4.6 \mathrm{um}$. The coordinates of the PPA point are $(-36,-87 \mathrm{um})$ for the FCO HD 1080p camera and (4-26 um) for FCO 720 P HD camera. In both cameras, the average values for the determined parameters practically coincide with the median values which allows us to conclude that there were no gross errors in the calibration process. From the analysis of the received data it can also be concluded that the internal orientation elements are stable.

To check the calibration process results from the PMS software, calibration was also performed using the TIMC software. Table 4 presents the differences in the internal orientation elements.

Table 4. Comparison of calibration results for the FCO HD 1080p camera and the FCO HD 720 camera obtained in the PMS and TIMC software

\begin{tabular}{cccccccc}
\hline \multicolumn{7}{c}{ FlyCam One HD 1080p } \\
\hline & $\mathbf{c}_{\mathbf{k}}[\mathbf{m m}]$ & $\mathbf{x}_{\mathbf{0}}[\mathbf{m m}]$ & $\mathbf{y}_{\mathbf{0}}[\mathbf{m m}]$ & $\mathbf{k}_{\mathbf{1}}$ & $\mathbf{k}_{\mathbf{2}}$ & $\mathbf{p}_{\mathbf{1}}$ & $\mathbf{p}_{\mathbf{2}}$ \\
\hline PMS & 10.693 & 2.965 & 1.600 & 0.002064 & -0.000099 & 0.000076 & -0.000350 \\
$\sigma$ & 0.016 & 0.04 & 0.009 & 0.000049 & 0.000006 & 0.000031 & 0.000046 \\
TIMC & 10.178 & 2.992 & 1.472 & 0.001321 & -0.000089 & -0.000169 & 0.000265 \\
$\sigma$ & 0.415 & 0.108 & 0.053 & 0.002148 & 0.000012 & 0.000006 & 0.000413 \\
$\Delta$ & 0.52 & -0.03 & 0.14 & 0.000744 & 0.000245 & -0.000614 & 0.000245 \\
\hline & & & \multicolumn{7}{c}{ FlyCam One HD720p } & & \\
\hline PMS & 7.980 & 3.003 & 1.661 & 0.003072 & -0.000292 & 0.000076 & -000035 \\
\hline$\sigma$ & 0.030 & 0.008 & 0.030 & 0.000049 & 0.000260 & 0.000067 & 0.000078 \\
TIMC & 7.744 & 2.711 & 1.496 & 0.002466 & -0.000171 & 0.000296 & 0.000245 \\
$\sigma$ & 0.215 & 0.161 & 0.982 & 0.001628 & 0.000201 & 0.000257 & 0.000039 \\
$\Delta$ & 0.54 & 0.29 & 0.16 & 0.000605 & -0.000120 & -0.000220 & -0.000595 \\
\hline
\end{tabular}

Analyzing the obtained differences it can be noted that the values obtained in the TimC have a greater error. It can also be noticed that there is a significant difference in the resulting values of $c_{k}$. It amounts to about $5 \%$ of the value of the focal length for the FCO1080camera and close to $7 \%$ for the FCO720p camera. The inaccuracy of determining this parameter may be caused by using $2 \mathrm{D}$ test.

The obtained results of the calibration process lead to the conclusion that the analyzed cameras can be used as imaging sensors on-board a UAV designed for cartometric purposes.

\section{Photographic aspect}

The next step in the calibration of these cameras is the photographic aspect.It is associated with the assessment of the quality of the obtained images by defining parameters which affect their photo-interpretational potential. These parameters include the ground resolved distance, the chromatic aberration and the fidelity of colour reproduction.

The photographic calibration had been carried out on the basis of a specialized ISO 12233 field test together with the Imatest 3.8 software.

The imagery data were obtained in laboratory conditions in 5 measurement series (each with different ISO) in 10 video sequences. After each sequence the camera was switched off and on again. Using the MTF 50 function, the GRD was defined in the horizontal direction $(\mathrm{X})$ and vertical $(\mathrm{Y})$. The results obtained are summarized in Table 4.

For the examined cameras, the resolution (on average) was practically at the same level (23.5 lp / mm FCO HD 1080P, $23 \mathrm{lp} / \mathrm{mm}$ FCO HD 720p). The largest errors were obtained for the resolution at ISO set to Auto.

The next step was to determine the value of the chromatic aberration. The value of the chromatic aberration is given as a percentage of the distance from the center of the image to the edge of the image. 
Table 5. Resolution of the FCO HD 1080p and FCO HD 720 p cameras

\begin{tabular}{ccccccccc}
\hline \multicolumn{9}{c}{ FCO HD 1080p } \\
\hline $\mathbf{I S O}$ & $\mathbf{X}[\mathbf{l p} / \mathbf{m m}]$ & $\boldsymbol{\sigma}$ & $\mathbf{Y}[\mathbf{l p} / \mathbf{m m}]$ & $\boldsymbol{\sigma}$ & $\mathbf{X}[\mathbf{l p} / \mathbf{m m}]$ & $\boldsymbol{\sigma}$ & $\mathbf{Y}[\mathbf{l p} / \mathbf{m m}]$ & $\boldsymbol{\sigma}$ \\
\hline $\mathbf{1 0 0}$ & 16.87 & 0.77 & 29.45 & 1.44 & 16.28 & 0.67 & 27.32 & 0.70 \\
$\mathbf{2 0 0}$ & 17.78 & 0.59 & 29.24 & 0.53 & 16.75 & 0.27 & 27.38 & 0.93 \\
$\mathbf{4 0 0}$ & 18.85 & 0.95 & 31.32 & 0.83 & 16.58 & 0.08 & 27.38 & 0.98 \\
$\mathbf{8 0 0}$ & 17.67 & 0.62 & 29.21 & 0.57 & 17.08 & 0.32 & 27.67 & 0.28 \\
$\mathbf{A U T O}$ & 17.52 & 0.71 & 27.76 & 1.45 & 17.53 & 0.89 & 29.48 & 1.09 \\
\hline
\end{tabular}

Figure 2 shows the values of chromatic aberration for the left and right edges of the frame as a function of the sensors set ISO value. The FCO HD 720P camera has an average chromatic aberration of $0.260 \%$ (the left edge $-0.336 \%, 0.183 \%$ right edge), which (according to the Imatest software manual) is very visible chromatic aberration. The FCO HD 1080P camera achieved slightly lower values of chromatic aberration equal to an average of $0.124 \%$ (the left edge $-0.148 \%$, the right edge of $0.1 \%$ ), which means a moderate aberration (visible at high magnification). The severity of the aberration on the right and left side of the frame (for both cameras) are significantly different from each other. The aberration on the right edge of the image is always smaller.

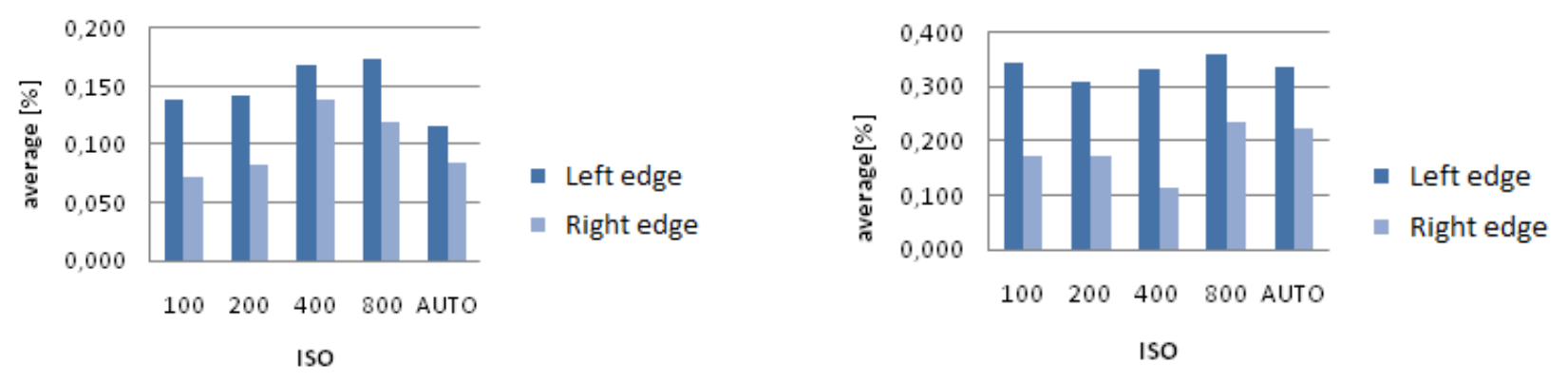

Fig. 2. The chromatic aberration value for FCOHD 1080p (above) and FCOHD720p (below)

\section{Determining GSD and swath width}

Additionally, in order to analyze the possibility of using a video camera as a UAV sensor, we looked at the way in which the GRD changes in relation to the flying altitude of the UAV on which it was mounted. Table 6 presents the GSD and the swath (A x B) as a function of altitude. As a reference, these same parameters calculated for the Sony Nex-5 camera have been added. This device is often used as a UAV sensor due to its small weight and the fact that it can be fitted with lenses with a fixed focal length (in this case $35 \mathrm{~mm}$ ).

Table 6. Comparison of GSD values for the FCO HD 1080p camera, FCO HD 720camera and NEX-5 camera as a function of altitude

\begin{tabular}{|c|c|c|c|c|c|c|c|c|c|}
\hline \multirow{3}{*}{$\underset{[\mathbf{m}]}{\mathbf{H}}$} & \multicolumn{3}{|c|}{ FCO HD 720p } & \multicolumn{3}{|c|}{ FCO HD 1080p } & \multicolumn{3}{|c|}{ SONY NEX $5\left[c_{k}=35 \mathrm{~mm}\right]$} \\
\hline & \multirow{2}{*}{ GSD [m] } & \multicolumn{2}{|c|}{ Range $[\mathrm{m}]$} & \multirow{2}{*}{$\begin{array}{c}\text { GSD } \\
{[\mathrm{m}]}\end{array}$} & \multicolumn{2}{|c|}{ Range $[\mathrm{m}]$} & \multirow{2}{*}{ GSD $[\mathrm{m}]$} & \multicolumn{2}{|c|}{ Range [m] } \\
\hline & & A & $\mathrm{B}$ & & A & $\mathrm{B}$ & & A & $\mathrm{B}$ \\
\hline 2 & 0.001 & 1.50 & 0.85 & 0.001 & 1.12 & 0.63 & 0.000 & 1.34 & 0.89 \\
\hline 5 & 0.003 & 3.76 & 2.11 & 0.001 & 2.80 & 1.58 & 0.001 & 3.34 & 2.23 \\
\hline 20 & 0.012 & 15.03 & 8.46 & 0.006 & 11.22 & 6.31 & 0.003 & 13.38 & 8.92 \\
\hline 30 & 0.018 & 22.56 & 12.69 & 0.009 & 16.83 & 9.47 & 0.004 & 20.06 & 1338 \\
\hline 40 & 0.023 & 30.07 & 16.92 & 0.012 & 22.44 & 12.62 & 0.006 & 26.75 & 1784 \\
\hline 50 & 0.029 & 37.59 & 21.15 & 0.015 & 28.05 & 15.78 & 0.007 & 33.44 & 2229 \\
\hline 90 & 0.053 & 67.67 & 38.06 & 0.026 & 50.49 & 28.40 & 0.013 & 60.19 & 40.13 \\
\hline 100 & 0.059 & 75.19 & 42.29 & 0.029 & 56.11 & 31.56 & 0.015 & 66.88 & 44.59 \\
\hline
\end{tabular}


In term sof a single frame swath, there is practically no difference between the FCO HD 720p and Nex-5 cameras. In addition to the aspects wchich had already been covered, an economical factor is surely in favour of the two cameras, with the frame sensor being overt 50\% more expensive than the FCO HD 1080p and over 70\% more costly in comparison to the FCO HD 720p.

Based on the recommended requirements about the performance of digital systems for mapping purposes it can be concluded that images obtained from a height of $100 \mathrm{~m}$ by the FCO HD 1080p camera will enable mapping on a scale of 1:2000 and smaller. In contrast, using the FCO HD 720p camera, this scale will be approximately 1:5000

\section{Conclusion}

Ultra lightweight UAVs are increasingly used as a platform for various remote sensing sensors. The majority are, however, cameras typically used to acquire imagery data in order to develop orthophotmaps. The alternative is a light video cameras that due to the high number of images obtained in a function of time, their low weight and relatively high resolution are ideal for remote sensing applications.

An additional advantage of video cameras is the fact that it is relatively simple to transmit video data to ground control stations where it can be processed. This allows you to conduct data analyses and obtain in quasi-real time.

The results of the calibrations presented in this article for the FCO HD 1080i and FCO HD 720i cameras confirm that these cameras can be successfully used as an imaging sensor. Video imagery data acquired from onboard a low-cost UAV can be used during the engineering inspections, for the development of rapid orthophotomaps of investment areas and for change analyses.

Lightweight video sensors in conjunction with "I low cost" UAV are undoubtedly an interesting combination that gives a lot of possibilities in terms of acquiring imaging data.

\section{Acknowledgements}

The present article is part of research work carried out in the "Innovative remote sensing system for the monitoring of pollutants in rivers, offshore waters and flooded areas" project - PBS1/B9/8/2012

\section{References}

[1] Bendig, J.; Bolten, A.; Bareth, G. 2012. Introducing a low-cost mini-UAV For thermal- and multispectral-imaging, in International Archives of the Photogrammetry, Remote Sensing and Spatial Information Sciences, Volume XXXIX-B1, 2012 XXII ISPRS Congress, 25 August - 01 September 2012, Melbourne, Australia.Gruen A., 2001. System calibration trough selfcalibration. Calibration and orientation of cameras in Computer Vision. Springer Series in Information Sciences 34: 163-194.

[2] Ganci, G.; Handley, H. 1998. Automation in videogrametry, Int. Archives of Photogrammetry and Remote Sensing 32(5): 117-132.

[3] Cronk, S.; Fraser, J.G.; Hanley, H. B. 2006. Automatic Calibration of Color Digital Cameras, Photogrametric Record Brown, D.C, 1971, Close range camera calibration. PE\&RS 37(8): 855-866.

[4] Fraser, J. 1997. Digital camera self calibration, ISPRS Journal of Photogrammetry and remote sensing 52: 149-159. http://dx.doi.org/10.1016/S09242716(97)00005-1

[5] Remondinio, F.; Fraser, C. 2006. Digital camera Calibration methods: Considerations and comparisons, IAPRS Volume XXXVI, Part 5, Drezno

[6] Luhmann, T.; Robson, S.; Kyle, S.; Harley, I. 2006. Close Range Photogrammetry. Whittles Publishing, Scotland UK, 114-124, 448-458. 